\title{
Spectral Modulations in a Picosecond OPO Based on a Chirped Quasi-Phase Matched Crystal
}

\author{
Guillaume Walter ${ }^{1}$, Jean-Baptiste Dherbecourt ${ }^{1}$, Jean-Michel Melkonian ${ }^{1}$, Myriam Raybaut ${ }^{1}$, Cyril Drag $^{2}$, \\ Antoine Godard ${ }^{1, *}$ \\ ${ }^{I}$ DPHY, ONERA, Université Paris Saclay, F-91123 Palaiseau, France \\ ${ }^{2}$ Laboratoire de Physique des Plasma, Ecole Polytechnique-CNRS-Univ Paris-Sud-UPMC, Université \\ Paris-Saclay, 91128 Palaiseau, France \\ *antoine.godard@onera.fr
}

\begin{abstract}
We investigate and model spectral modulations that are specific features of OPOs based on chirped quasi-phase matched crystals. Their occurrence is related to cascaded three-wave mixing processes that are quasi-phase matched at different positions..

OCIS codes: (190.4970) Parametric oscillators and amplifiers, (190.4223) Nonlinear wave mixing, (190.4410) Nonlinear optics, parametric processes
\end{abstract}

Aperiodic-or chirped-quasi-phase-matching (QPM) nonlinear crystals offer unique features compared to conventional quadratic nonlinear materials. Indeed, one can engineer the phase-matched wavelengths as functions of the position $\mathrm{z}$ along the propagation axis in the material, the QPM grating period $\Lambda(\mathrm{z})$ being a function of $\mathrm{z}$.

Used as the amplification medium in an optical parametric oscillator (OPO), a chirped QPM crystal can provide a broad parametric gain spectrum without the need to use short crystals with reduced conversion efficiency. Such OPOs based on chirped QPM were reported in various temporal regimes [1-3].

In picosecond synchronously pumped OPOs (SPOPOs) based on chirped QPM crystals and broadband mirrors (without intracavity filter), we observe signal spectra consisting of a main peak with lower side lobes [4]. These spectral modulations are not observed for similar picosecond SPOPOs based on uniform QPM crystals.

Here, we report on the experimental and numerical investigations of these spectral modulations in OPOs based on chirped QPM, which still need to be explained. We show that the spectral modulations are induced by cascaded three-wave mixing between different parts of the interacting-waves spectra, quasi-phase matched at different positions in the nonlinear crystal.

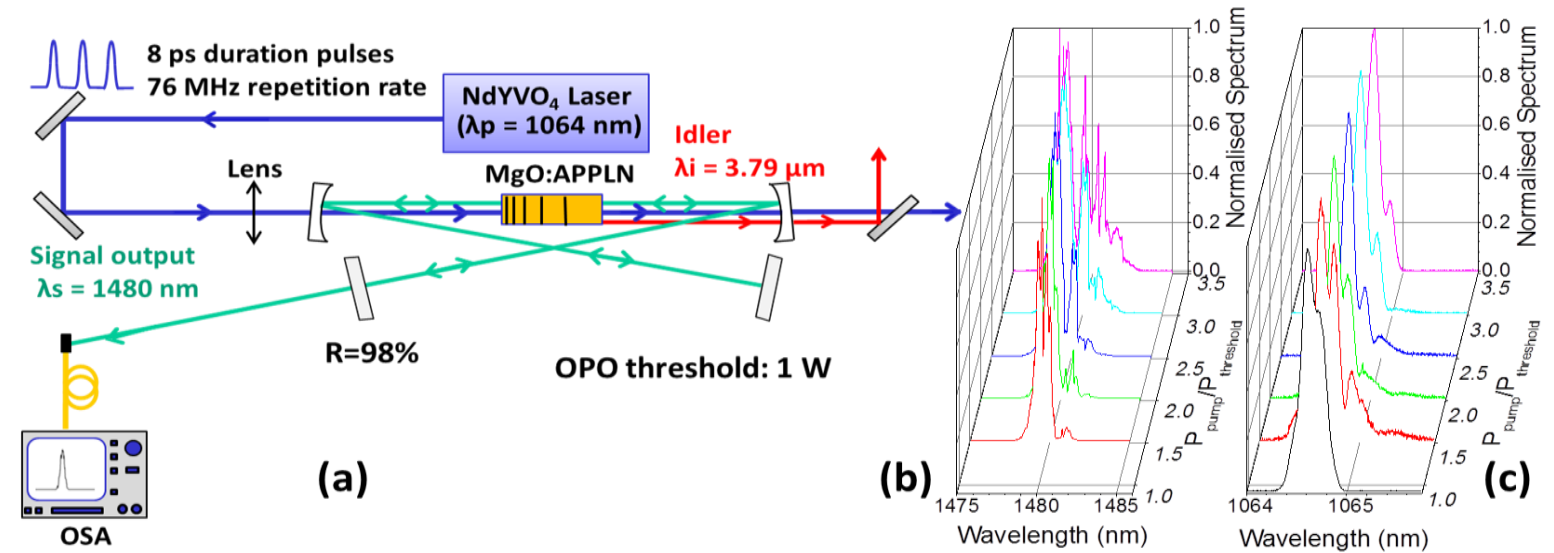

Fig. 1. Schematic diagram of the SPOPO setup (a). Evolution of the signal spectrum (b), and of the pump spectrum (c), as a function of the pump power.

As presented in Fig. 1(a), the SPOPO is based on an aperiodically poled lithium niobate (APPLN) crystal (HC Photonics) and is pumped by a Nd: $\mathrm{YVO}_{4}$ laser with a repetition rate of $76 \mathrm{MHz}$ and a pulse duration of 8 ps. The signal oscillating in the OPO cavity can be analyzed at the output of a plane mirror with a reflectivity of $98 \%$. Here, experiments are carried out with an apodized chirped QPM grating, with a central chirp rate of $1.17 \times 10^{4} \mathrm{~m}^{-2}$, leading to a parametric gain bandwidth of $40 \mathrm{~nm}$ and an oscillation threshold of $1 \mathrm{~W}$.

By the use of an optical spectrum analyzer (OSA), the pump and signal spectra can be studied. Fig. 1(b) and Fig. 1(c) present the evolution of the spectra of the signal and the depleted pump at the output of the nonlinear crystal, as functions of the pump power. The higher is the pump power, the broader is the emitted signal spectrum with more and more side lobes. In the same time, modulations are also observed in the depleted pump spectrum. 

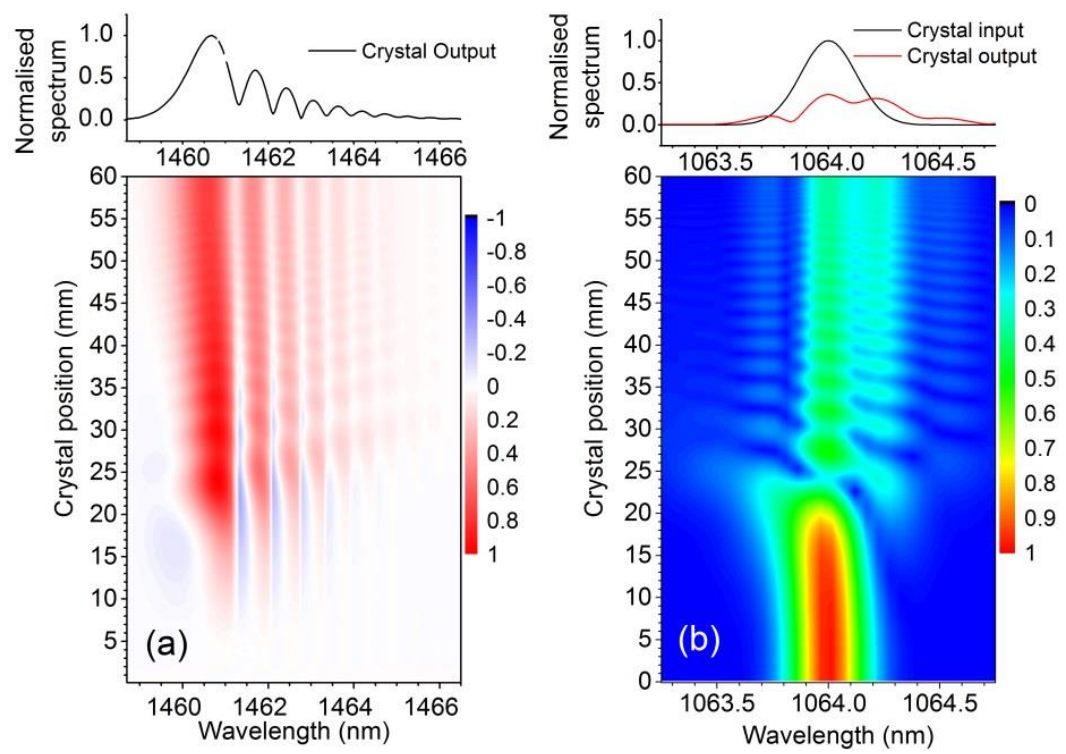

Fig. 2. Simulation at steady state of the parametric generation evolution of the signal spectrum (a), and of the evolution of the pump spectrum (b), through the APPLN crystal

A previously validated home-made numerical code has been used to understand the development of such modulations [3]. For that purpose, we model the evolution of the interacting parametric waves while they pass through the nonlinear crystal. Fig. 2 shows the evolution the signal and pump spectra during a propagation through the APPLN crystal when the OPO is in steady-state. From this figure, we can identify that wavelengths located either on peaks or on valleys of the output signal spectrum experience very different evolution while they propagate through the crystal. Indeed, the part of the spectrum which corresponds to modulation peaks experiences an exponential growth over a characteristic length, as expected from theory [5]. Then, this part has an oscillatory evolution through the rest of the crystal. The part which corresponds to modulation valleys of the spectrum exhibits a different evolution. These wavelengths first experience a power decrease before being amplified. As observed in Fig. 2(b), this decrease corresponds to the generation of new spectral sidebands in the traveling pump spectrum where signal spectrum modulation valleys exhibit a power decrease.

Such a behavior is consistent with cascaded difference- and sum-frequency generation (DFG-SFG) conversion: idler wavelengths are generated by quasi-phase-matched DFG between incident signal and pump pulses, then quasiphase-matched SFG occurs at other positions in the crystal between the generated idler and other signal wavelengths which generate pump sidebands. Such cascaded processes are enabled by the variation of the QPM period over the length of the aperiodic nonlinear crystal.

The origin of the spectral modulations in OPOs based on chirped QPM crystals are thus explained for the first time owing to our study. Further details, additional experimental and numerical results will be presented.

\section{Funding}

Agence Nationale de la Recherche (ANR) (ANR-14-CE26-0036); "Investissements d'Avenir" LabEx PALM (SYCLOP and SORA) (ANR-10-LABX-0039).

\section{References}

[1] K. A. Tillman, D. T. Reid, D. Artigas, J. Hellström, V. Pasiskevicius, and F. Laurell, "Low-threshold, high-repetition-frequency femtosecond optical parametric oscillator based on chirped-pulse frequency conversion," J. Opt. Soc. Am. B 20, 1309-480 (2003).

[2] K. A. Tillman, D. T. Reid, "Monolithic optical parametric oscillator using chirped quasi-phase matching,” Opt. Lett. 32, 1548-1550 (2007).

[3] D. Descloux, C. Laporte, J.-B. Dherbecourt, J.-M. Melkonian, M. Raybaut, C. Drag, and A. Godard, "Spectrotemporal dynamics of a picosecond OPO based on chirped quasi-phase-matching," Opt. Lett. 40, 280-283 (2015) and erratum in Opt. Lett. 43 , 494 (2018).

[4] D. Descloux, G. Walter, E. Cadiou, J.-B. Dherbecourt, G. Gorju, J.-M. Melkonian, M. Raybaut, C. Drag, and A. Godard, "Wide and fast dispersion tuning of a picosecond OPO based on aperiodic quasi-phase matching using an axially chirped volume Bragg grating," Opt. Lett. 41, 4060-4063 (2016)

[5] M. Charbonneau-Lefort, B. Afeyan, and M. M. Fejer, "Optical parametric amplifiers using chirped quasi-phase-matching gratings $\{$ I $\}$ : practical design formulas,” J. Opt. Soc. Am. B 25, 463-480 (2008). 